\title{
ORIGINAL ARTICLE \\ Discrepancies between clinical assessments of sensory function and electrical perceptual thresholds after incomplete chronic cervical spinal cord injury
}

\author{
RA Macklin ${ }^{1,3}$, VJ Brooke ${ }^{1,3}$, FJ Calabro ${ }^{1}$, PH Ellaway ${ }^{2}$ and MA Perez ${ }^{1}$
}

\begin{abstract}
Study design: Prospective experimental.
Objectives: To compare sensory function as revealed by light touch and pin prick tests of the International Standards for Neurological Classification of Spinal Cord Injury (ISNCSCI) and the electrical perceptual threshold (EPT) exams in individuals with chronic incomplete cervical spinal cord injury (SCI).
\end{abstract}

Setting: Pittsburgh, United States.

Methods: EPT was tested using cutaneous electrical stimulation $(0.5 \mathrm{~ms}$ pulse width, $3 \mathrm{~Hz})$ in 32 healthy controls and in 17 participants with SCl over key points on dermatomes C2 to T4 on each side of the body. Light touch and pin prick ISNCSCl scores were tested at the same key dermatomes in SCl participants.

Results: In controls, EPT values were higher in older males $(1.26 \pm 0.2 \mathrm{~mA}$, mean \pm s.d.) compared with younger males $(1.0 \pm 0.2 \mathrm{~mA})$ and older females $(0.9 \pm 0.2 \mathrm{~mA})$, regardless of the dermatome and side tested. Fifteen out of the seventeen $\mathrm{SCl}$ participants showed that the level of sensory impairment detected by the EPT was below the level detected by the ISNCSCI (mean $=4.5 \pm 2.4$, range 1-9). The frequency distribution of EPTs was similar to older male controls in dermatomes above but not below the ISNCSCI sensory level. The difference between EPT and ISNCSCI sensory level was negatively correlated with the time post injury.

Conclusions: The results show that, in the chronic stage of cervical SCI, the EPT reveals spared sensory function at lower ( 5) spinal segments compared with the ISNCSCI sensory exam. It is hence found that the EPT is a sensitive tool to assess recovery of sensory function after chronic $\mathrm{SCl}$.

Spinal Cord (2016) 54, 16-23; doi:10.1038/sc.2015.104; published online 30 June 2015

\section{INTRODUCTION}

There are $\sim 12000$ new cases of spinal cord injury (SCI) every year in the United States, with an estimated 280000 persons currently living with SCIs. About $66 \%$ of all new injuries are classified as incomplete, and $45 \%$ of these affect the cervical spinal cord. Incomplete cervical SCI impairs somatic sensory function, which markedly diminishes the quality of life. ${ }^{1}$ A major role of somatic sensory receptors is to provide information about the state of the motor system to allow performance of complex movements and behaviors. Thus, the assessment of sensory function is critical to examine the degree of dysfunction and spontaneous or therapymediated recovery after SCI.

The current gold standard for assessing light touch and pin prick in humans with SCI is the International Standards for Neurological Classification of Spinal Cord Injury (ISNCSCI ${ }^{2}$ ). The ISNCSCI has been extensively used to assess individuals immediately after the injury and longitudinally. ${ }^{3}$ However, because the results are variable and the recording system itself does not capture detailed information about sensory function there is a need for quantitative sensitive outcome measurements of sensory function that supplement the ISNCSCI. ${ }^{4-6}$ There are currently 679 SCI clinical trials in various phases of research (ClinicalTrials.gov), supporting the need for better assessment tools for SCI translational research.

The electrical perceptual threshold (EPT) exam measures the sensory threshold or minimally detectable electrical stimulus intensity applied to the skin. It was initially used to monitor peripheral nerve function ${ }^{7}$ and then adapted to be used in the same key dermatome points as used in the ISNCSCI $^{8}$ for individuals with SCI. ${ }^{9-13}$ At present, all studies that used the EPT in participants with SCI detected sensory deficits as compared with healthy controls. The EPT is a reliable exam across examiners and has sufficient sensitivity to assess sensory function across multiple sessions. ${ }^{10,13}$ So far studies using the EPT involved heterogeneous groups including individuals in the acute and chronic phase of SCI with complete and incomplete injuries at the cervical, thoracic and lumbar spinal cord. When the EPT results were directly compared with the pin prick and light touch sensory portions of the ISNCSCI exam, it was found that the sensory level detected by the EPT was either higher, lower or at the same level as the

${ }^{1}$ Department of Physical Medicine and Rehabilitation, Center for the Neural Basis of Cognition, Systems Neuroscience Institute, University of Pittsburgh, Pittsburgh, PA, USA and ${ }^{2}$ Department of Medicine, Division of Brain Sciences, Imperial College, London, UK

${ }^{3}$ These authors contributed equally to the work.

Correspondence: Dr MA Perez, Department of Neurological Surgery, The Miami Project to Cure Paralysis, Lois Pope Life Center, 1095 NW 14th Terrace, Room 1-28, Mail Locator R-48, University of Miami, Miami, FL 33136, USA.

E-mail: perezmo@miami.edu

Received 4 February 2015; revised 11 May 2015; accepted 19 May 2015; published online 30 June 2015 
ISNCSCI, ${ }^{9,11,13}$ which may result from the heterogeneity of the test group. Studies that compare the sensory level detected by the EPT versus ISNCSCI in a more homogenous SCI group are needed to reveal specific sensory impairments after SCI.

The purpose of our study was to compare sensory function as revealed by the ISNCSCI and EPT exams in a group of participants with chronic ( $\geqslant 1$ year) incomplete cervical SCI. Studies have reported that individuals with chronic cervical incomplete SCI have the ability to improve upper-limb sensorimotor function. ${ }^{14}$ We expected that, after chronic incomplete cervical SCI, the EPT would be more sensitive in detecting spared sensory function at or below the sensory level determined by the ISNCSCI exam.

\section{MATERIALS AND METHODS}

\section{Subjects}

Seventeen participants with SCI (mean age $=52.5 \pm 15.1$ years, 2 females; Table 1) and 32 age-comparable controls (mean age $=46.1 \pm 20.1$ years, $P=0.23,16$ females) participated in the study. All subjects gave informed consent to experimental procedures, which were approved by the local ethics committee at the University of Pittsburgh. Previous studies in healthy controls demonstrated differences across age and gender in EPT values. ${ }^{15}$ Therefore, healthy controls were divided into gender and age-comparable categories: younger males and females (younger females, mean age $=25.4 \pm 3.9$ years, range $20-32, n=8$; younger males, mean age $=29.8 \pm 9.8$ years, range $20-50$, $n=8 ; P=0.21$ ) and older males and females (older females, mean age $=64.0$ \pm 7.2 years, range $53-72, n=8$; older males, mean age $=65.1 \pm 6.6$ years, range $54-77, n=8, P=0.74)$. Participants with SCI had a chronic ( $\geqslant 1$ year), cervical injury $(\mathrm{C} 1-\mathrm{C} 8)$ and incomplete SCI as determined by the American Spinal Injuries Association Impairment Scale (AIS) grade. Light touch and pin prick were measured using the ISNCSCI sensory scores. Two out of the seventeen SCI participants were categorized as AIS B and the other fifteen were classified as incomplete AIS C and D (Table 1).

\section{EPT}

Testing was conducted using constant current square wave electrical pulses ( $0.5 \mathrm{~ms}$ pulse width duration, $3 \mathrm{~Hz}$ stimulation frequency, DS7A, Digitimer Ltd,
Hertfordshire, UK). Stimuli were delivered to the skin over the ISNCSCI sensory key points in 22 dermatomes between C2 and T4 (Figure 1) on both sides of the body by using disposable adhesive electrodes. The cathode was positioned over the ISNCSCI sensory key point, and the anode was placed on the ipsilateral arm of the applied stimulus. The stimulus intensity was manually increased in increments of $0.1 \mathrm{~mA}$ up to $10 \mathrm{~mA}$. Each subject was given a familiarization trial run in order to recognize the electrical pulses. Subjects were asked to report verbally when the first sensation was felt. The procedure was repeated three times on each dermatome, and the EPT $(\mathrm{mA})$ was calculated as the mean of the intensities when sensation just disappeared on each trial (lowest descending stimulus intensity). The perceived stimulus was described as a light 'tapping' or gentle 'pulsing' sensation and was not reported as painful by any of the subjects. Subjects were blind to the amplitude of the stimulus current. In healthy controls, the mean EPT values and values 2 s.d. above the mean were calculated at each dermatome. Frequency histograms were created for groups separated by gender and age (Figure 3). In SCI participants, EPTs were analyzed in two ways. First, as in controls, the mean EPT value was calculated and values 2 s.d. above the mean for each dermatome. EPT was considered 'abnormal' when it was $>2$ s.d. of the mean value of an age and gender comparable control group. Second, we assessed significant deviations of EPT values in SCI participants from the mean results in age and gender comparable controls using $\mathrm{Z}$-scores. A Z-score represents the distance between the raw score and the population mean in units of the standard deviation. Thus, a Z-score is negative when the raw score is below the mean and positive when it is above. Frequency histograms were created for old males and SCI participants in dermatomes located above and below the ISNCSCI sensory level. EPT was completed on two separate occasions separated by $1-3$ weeks in healthy controls and in individuals with SCI to examine reliability of measurements across sessions.

\section{ISNCSCI}

Individuals with SCI participated in a neurological assessment from a spinal cord physician, following the standards by the ISNCSCI guidelines. ${ }^{2}$ The ISNCSCI exam involved sensory and not motor assessment. The sensory exam included the pin prick and light touch components from dermatomes $\mathrm{C} 2$ to T4 on both sides of the body (Figure 1 and Table 1). ISNCSCI sensory examinations were completed by the same physician on two separate occasions separated by $1-3$ weeks in SCI participants to examine reliability of

Table 1 Spinal cord injury participants

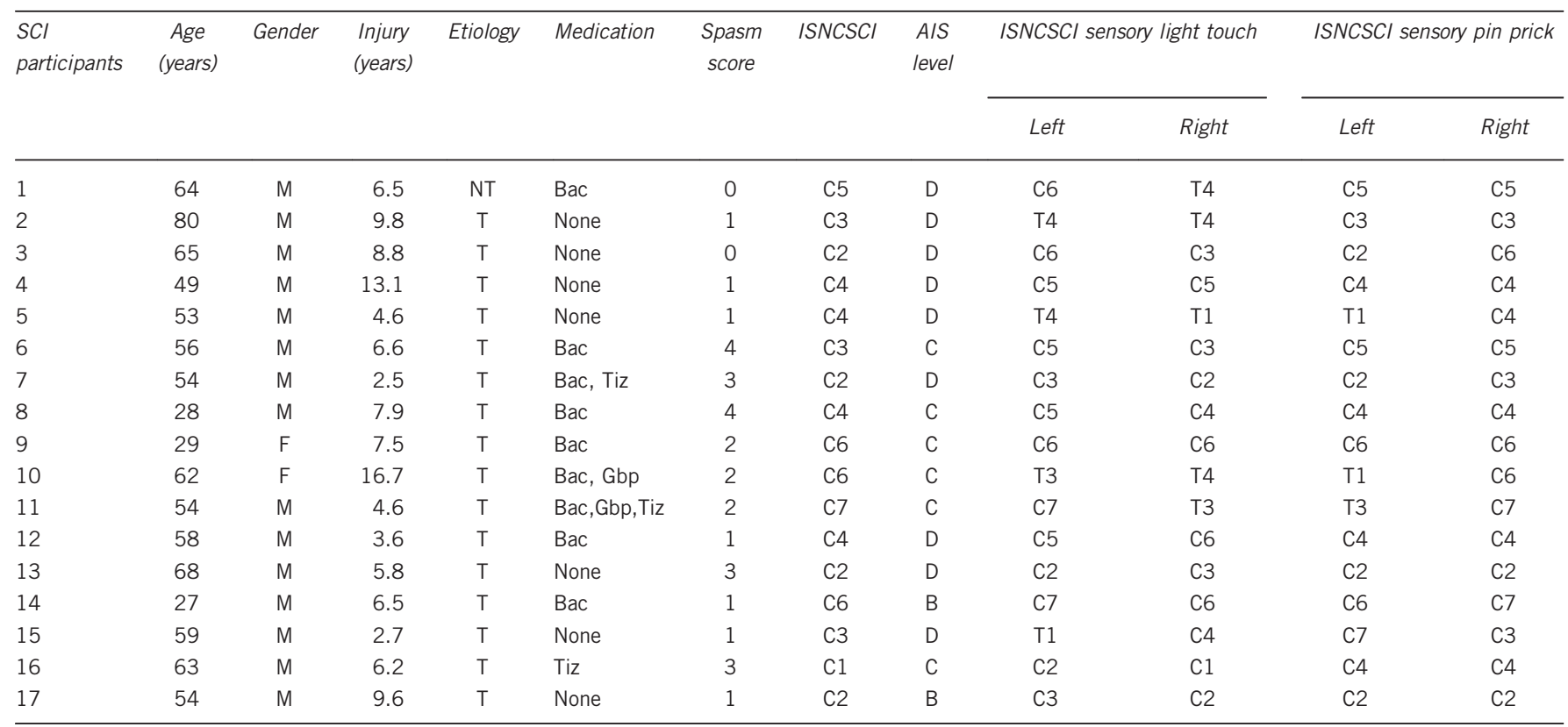

Abbreviations: AIS, American Spinal Injuries Association Impairment Scale; Bac, baclofen; F, female; Gbp, gabapentin; ISNCSCI, International Standards for Neurological Classification of Spinal Cord Injury; M, male; NT, non-traumatic; SCI, spinal cord injury; T, traumatic; Tiz, tizanidine.

Spasm frequency score: $0=$ no spasms, $1=$ one or fewer spasms per day, $2=1$ to 5 spasms per day, $3=5$ to 10 spasms per day, and $4=10$ or more spasms per day. 


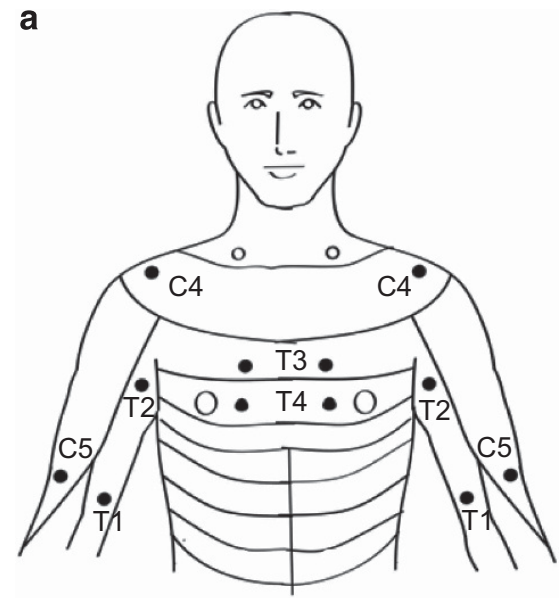

b

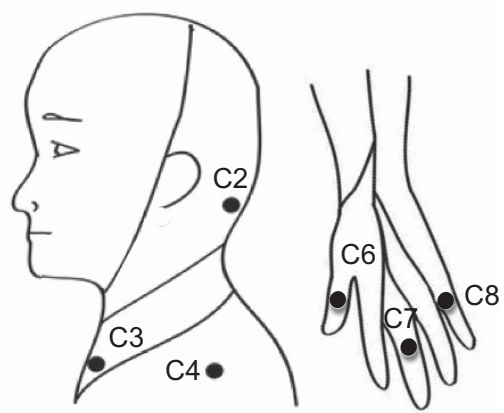

Figure 1 Experimental Setup. Sensory key points by spinal dermatomes reproduced from the International Standards for Neurological Classification of Spinal Cord Injury (ISNCSCI). a) C4,C5,T1-T4; b) C2-C4 and C6-C8. The ISNCSCl key points in dermatomes from C2 to T4 were tested in the study.

measurements across sessions. Note that the ISNCSCI sensory level was defined as the most caudal intact dermatome for both pin prick and light touch sensation. Therefore, there may be some sensation, although abnormal, below the ISNCSCI sensory level.

\section{RESULTS}

Repeated-measures analyses of variance were performed to determine the effect of gender (female, male), age (young, old), side (left, right) and dermatome ( $\mathrm{C} 2$ to $\mathrm{T} 4,11$ dermatomes per side) on EPT values in healthy controls by using the dermatome as the repeated measure. Bonferroni post hoc analysis was used to test for significant comparisons. Paired $t$-tests were performed to test the difference between groups and sides as needed. Z-scores for the SCI population were computed for each dermatome by using the following formula: (SCI EPT value-EPT mean value for age and gender comparable population)/(s.d. for same age and gender comparable population). Testretest rater reliability was measured by calculating intra-class correlation coefficients (ICCs). ICCs performed on the sensory level as assessed by the EPT showed good reliability (ICC $=0.92,95 \%$ confidence interval $=0.85-0.96$ ). ICCs performed on the sensory level as assessed by the ISNCSCI showed lower reliability compared with EPT $(\mathrm{ICC}=0.62,95 \%$ confidence interval $=0.1-0.87$ ). A Pearson correlation analysis was used as needed. Significance was set at $P<0.05$. Group data are presented as the means \pm s.d. in the text.

\section{EPT: healthy controls}

Figure 2 illustrates the group mean EPTs for all dermatomes tested in healthy controls separated by gender and age. Note that EPT values were higher in males compared with females, regardless of the side and dermatome tested (Figure 2a). Also, note that older males showed higher EPT values compared with younger males and older females, regardless of the side and dermatome tested (Figures $2 \mathrm{c}$ and e).

Repeated-measures analysis of variance showed a significant effect of gender $\left(\mathrm{F}_{(1,28)}=25.9, P<0.001\right)$, age $\left(\mathrm{F}_{(1,28)}=6.7, P=0.01\right)$ and their interaction $\left(\mathrm{F}_{(1,28)}=6.3, P=0.01\right)$ but not of side $\left(\mathrm{F}_{(1,28)}=0.9\right.$, $P=0.43)$ on EPT values. Post hoc testing showed that, when we separated subjects by gender and age, old males had a higher threshold compared with young $(P<0.001$; Figure $2 \mathrm{c})$, whereas no differences were found between old and younger females $(P=0.95$; Figure $2 \mathrm{~d})$. Old males had a higher threshold compared with old females $(P<0.001$; Figure 2e), but these differences were not present between young males and females $(P=0.10$; Figure $2 \mathrm{f})$.

Figure 3 illustrates frequency histograms using EPT values in all control subjects. We found that the frequency distribution of EPTs in females and young individuals was shifted to the left compared with males and old individuals, suggesting that in most of the dermatomes tested EPT values were lower in females compared with males (Figure 3a) and in young vs old subjects (Figure 3b). The differences between gender and age became clear by our finding that old males have a frequency distribution of EPT values shifted to the right compared with younger males (Figure 3c) and older females (Figure 3d). Note that because EPTs varied significantly based on age and gender, our SCI participants (age $=52.5 \pm 15.1$ years) were most of the time compared with older male controls (age $=64.0 \pm 7.2$ years, $P=0.11$ ), as this constituted the largest group of SCI participants.

\section{EPT: SCI participants}

Figure 4 illustrates EPT values and their subsequent Z-score analysis in three representative individuals. Note in SCI participant \#1 (Table 1 and Figure 4a), the EPT exam showed a unilateral sensory impairment on the right side below T2. The level of sensory impairment detected by the EPT was located five segments below the ISNCSCI sensory level as shown by the difference between the black and gray lines. In agreement, the Z-score analysis revealed a significant deviation of EPT values from the mean of old male controls below T2 on the right side. In SCI participant \#2 (Table 1 and Figure 4b), the EPT results showed a bilateral symmetric sensory impairment below dermatome $\mathrm{C} 4$. The sensory level detected by the EPT was located one segmental level below the ISNCSCI sensory level. Similarly, the Z-score analysis revealed significant bilateral deviations of EPT values from the mean of old male controls below C4 bilaterally. Note that both individuals showed normal light touch scores several segments below the ISNCSCI sensory level (Table 1), highlighting the lack of sensitivity of the ISNCSCI exam. In SCI participant \#3 (Table 1 and Figure 4c), the EPT and Z-scores revealed significant bilateral asymmetric deviations of EPT values from the mean of old male controls from C8 on the left and from C6 on the right side. The EPT sensory level was five segments below the ISNCSCI sensory level on the left side and three segments below on the right side.

Figure 5 illustrates the EPT and ISNCSCI sensory level in all SCI subjects tested. Note that in the majority of SCI participants the EPT sensory level was found several segments below the ISNCSCI sensory level, regardless of the side tested (left side 14/17, right side 15/17). On average, the EPT indicated a sensory level $4.5 \pm 2.4$ segments below that detected by ISNCSCI exam (range 1-9). In one SCI participant, the EPT sensory level was one segment higher than the ISNCSCI sensory level in one of the sides tested, and in another participant with SCI the EPT was the same as the ISNCSCI sensory level in both sides. We also found that the frequency distribution of 
a

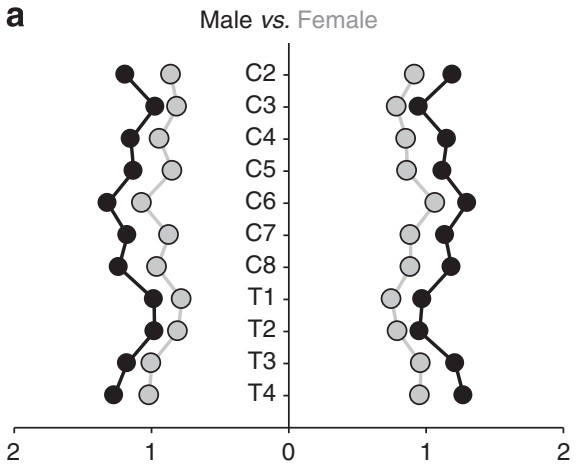

C

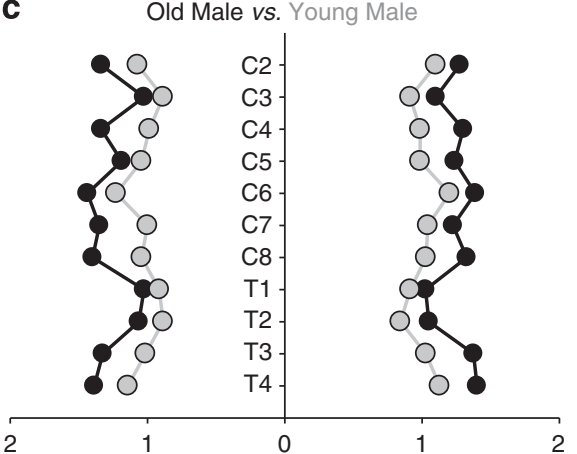

Old Male vs. Old Female

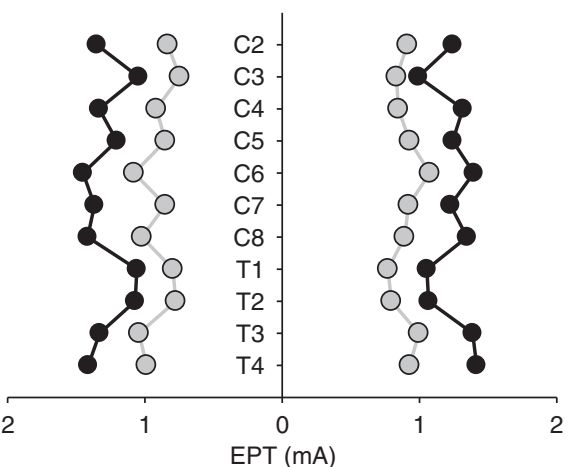

b Old vs. Young

Old vs. Young

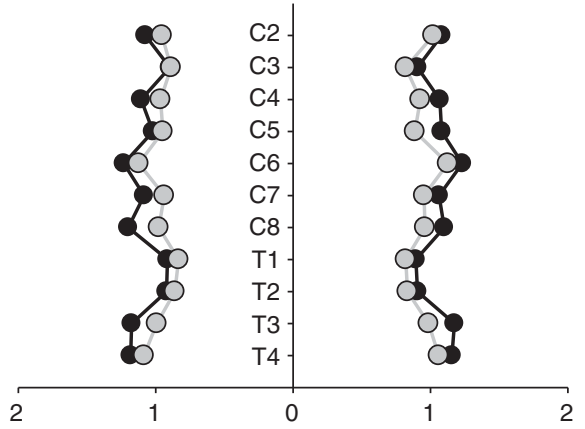

d

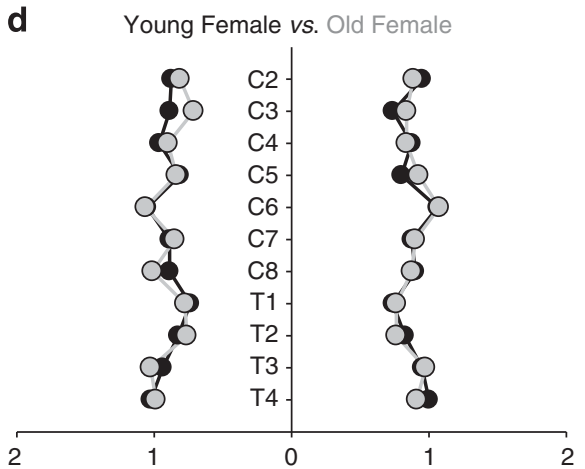

f

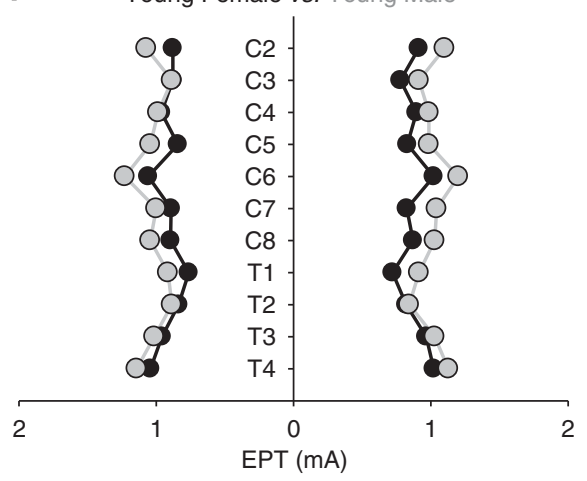

Figure 2 EPT — healthy controls. EPT results plotted by gender (male, black circles vs female, gray circles; a) and age (young, $\leqslant 50$ years old, gray circles vs old, $\geqslant 50$ years old, black circles; b) in all healthy controls tested. Data for old and young males (black and gray circles; c), young and old females (black and gray circles; d), old males and females (black and gray circles; e) and young females and males (black and gray circles; f) are also presented. The $x$-axis shows the stimulus intensity (mA) used during testing, and the $y$-axis shows the dermatomes tested. Note that overall older males show higher EPT values.

EPTs was similar between SCI participants and old male controls in dermatomes located above the sensory level but shifted to higher values, wider and of smaller amplitude compared with controls in dermatomes located below the sensory level, regardless if the sensory level was detected by the ISNCSCI (Figure 6a) or EPT (Figure 6b) exams. A negative correlation was found between differences found between EPT and ISNCSCI sensory level and the time post SCI $(r=-0.73, P<0.001$; Figure 7$)$. Thus, participants with longer times with SCI showed a smaller difference between EPT and ISNCSCI sensory level.

\section{DISCUSSION}

Our results demonstrate that, in the chronic stage of cervical SCI, the EPT exam reveals spared sensory function at lower $(\sim 5)$ spinal segments compared with the ISNCSCI. The frequency distribution of EPTs in SCI participants was similar to old male controls in dermatomes above but not below the ISNCSCI sensory level. The difference between EPT and ISNCSCI scores was negatively correlated with the time post injury: individuals who had SCI for longer time showed smaller differences between the sensory level detected by the EPT and ISNCSCI. Thus, our findings indicate that the EPT is a sensitive tool to assess recovery of sensory function after chronic human SCI.

\section{EPT vs ISNCSCI sensory level}

Our results in SCI participants agree with previous studies showing that the EPT exam is sensitive to detect sensory deficits in the SCI population. ${ }^{9-13}$ We demonstrate for the first time that, after chronic 
a

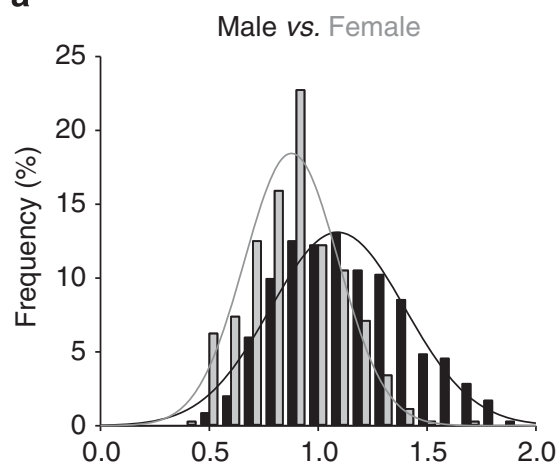

C

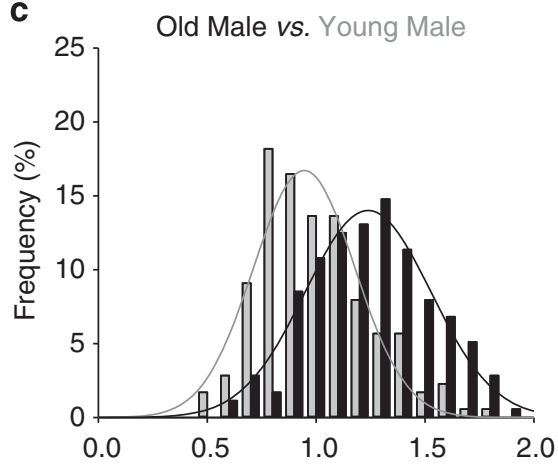

e

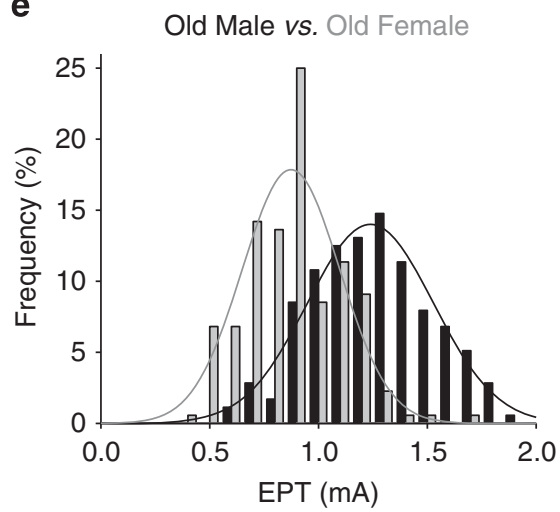

b

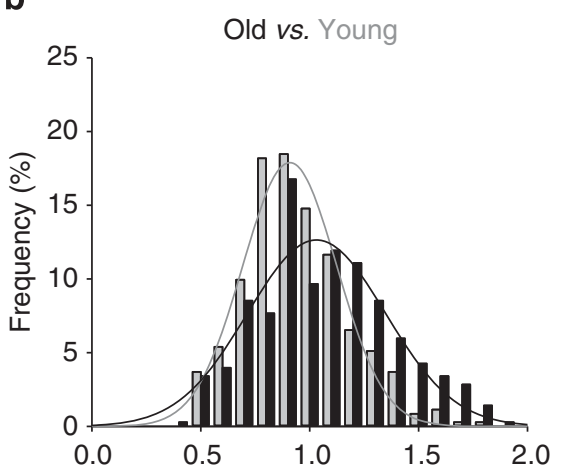

d

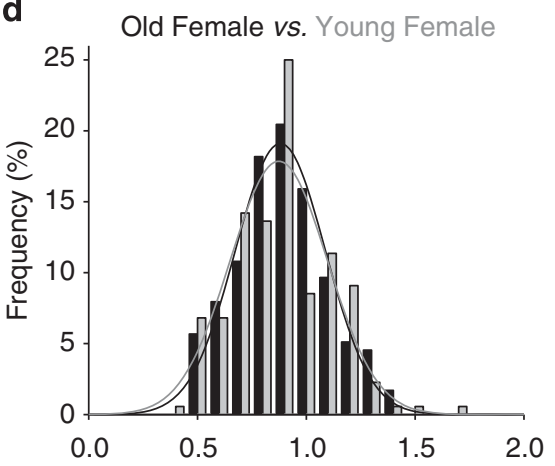

f

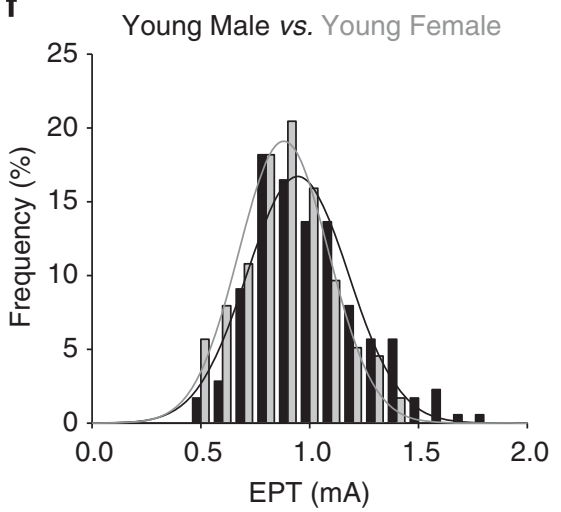

Figure 3 EPT frequency histograms. In the graphs, the abscissa shows the stimulus intensity (mA) used during testing and the ordinate shows the number of times that the intensities were repeated, regardless of the dermatome tested expressed as a \%. The fitted lines show the frequency distribution for males and females (black and gray bars; a), old and young individuals (black and gray bars; b), old and young males (black and gray bars; c), old and young females (black and gray bars; d), old males and females (black and gray bars; e) and young males and females (black and gray bars; f). Note that the frequency distribution of EPT values in older males was shifted to the right compared with young males and old females.

incomplete cervical SCI, the most caudal unaffected sensory level detected by the EPT can be $\sim 5$ segments below those detected by the ISNCSCI. Previous studies comparing the EPT results with the pin prick and light touch sensory portions of the ISNCSCI exam found that the EPT sensory level was either higher, lower or at the same level as the ISNCSCI in individuals with different characteristics and degrees of SCI. ${ }^{9-13}$ We now show in a more homogeneous group of SCI participants that the EPT can detect changes below the ISNCSCI exam in the majority of individuals. Indeed, in contrast to the acute phase where the sensory level measured by the EPT was typically several segments above the clinical ISNCSCI sensory level, ${ }^{13}$ our results showed that in the chronic phase of SCI the EPT detects changes below the clinical exam level. This in part might be related to differences in physiological and behavioral processes taking place in the acute or chronic phases of SCI. ${ }^{16}$ A possibility is that in the acute phase of SCI the EPT might be more sensitive to detect small sensory impairments that were missed by the ISNCSCI exam, whereas in the chronic phase the consistently lower EPT values suggest that this exam is more sensitive to assess the extent of recovery. This is in agreement with previous results in individuals with incomplete SCI showing that the number of participants with a sensory level detected by EPT below the level assessed by the ISNCSCI exam was larger 6 months compared with 1 month after the injury. ${ }^{13}$ This is also supported by the inverse correlation that we found between differences in EPT and the ISNCSCI sensory level and time post SCI. Here, individuals with SCI for longer periods of times were those who showed smaller differences between the sensory level detected by the EPT and ISNCSCI. 
a
SCl participant \#1

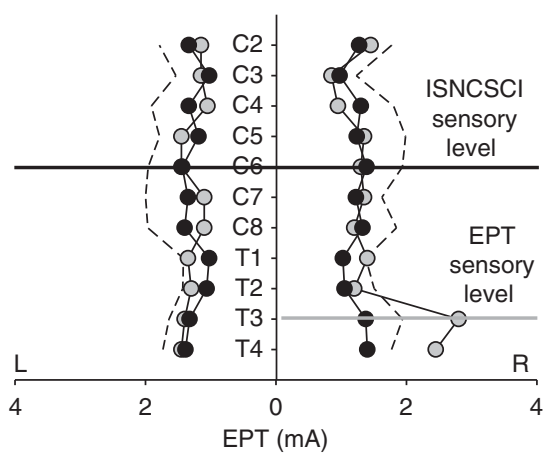

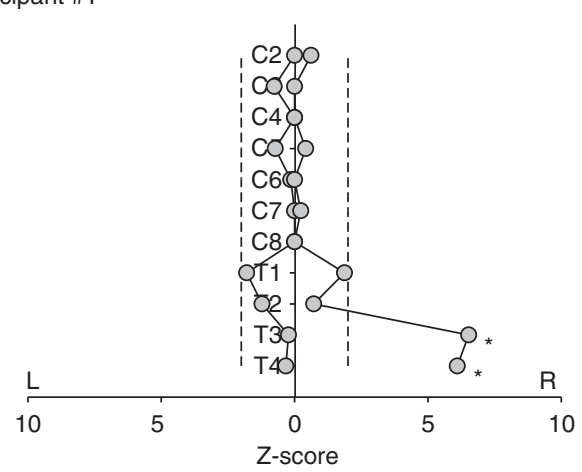

SCI participant \#2 b

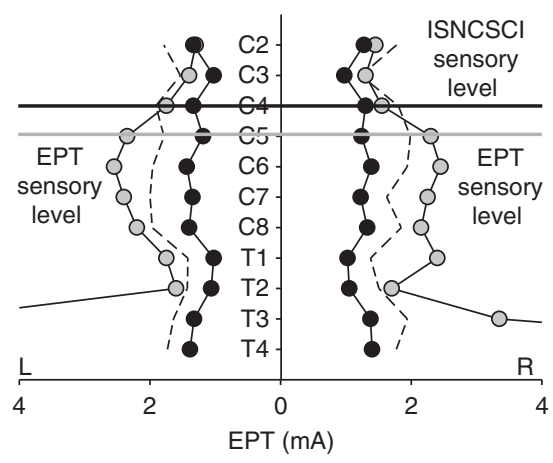

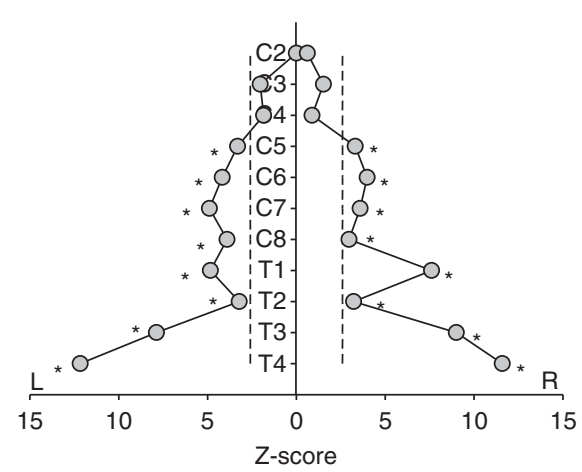

SCI participant \#3

C

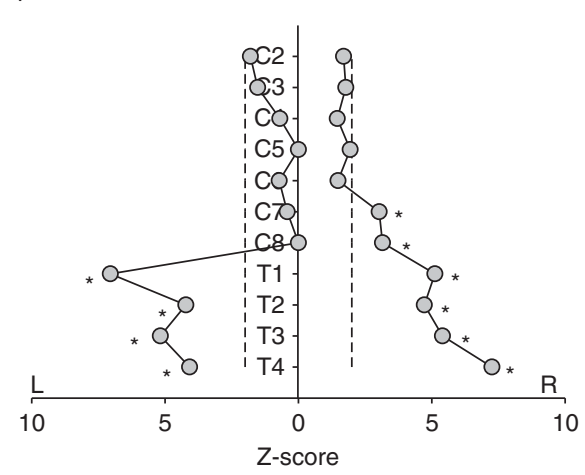

Figure 4 EPT vs ISNCSCI in SCl participants. EPT values and Z-score are shown in representative SCl participants (gray circles) compared with agecomparable old males (black circles). Dotted lines represent values 2 s.d. from the mean of male controls. ISNCSCl sensory level is shown by a horizontal black line, and the EPT level is shown by a horizontal gray line. Note: in $\mathrm{SCl}$ participant \#1 (a), the EPT and Z-scores showed a unilateral sensory deviation on the right side below T2. In SCl participant \#2 (b), the EPT and Z-scores showed a bilateral symmetric sensory deviation below dermatome C4, whereas in SCI participant \#3 (c) the EPT and the Z-score analysis revealed significant bilateral asymmetric deviations of EPT values from the mean of old male controls below $\mathrm{C} 8$ on the left and below $\mathrm{C} 6$ on the right side. ${ }^{*} P<0.05$.

A disagreement between EPT and ISNCSCI sensory level was seen in $\sim 90 \%$ of the SCI participants in at least one of the sides of the body. A possibility is that differences in the sensory fibers targeted by both examinations affected our results. The EPT exam mainly assesses dorsal column function, ${ }^{9}$ whereas the ISNCSCI sensory exam is widely accepted as assessing dorsal column function in the light touch test and the anterolateral spinothalamic tract in the pin prick test, ${ }^{16}$ despite the fact that the pin prick test involves a strong element of discrimination (between sharp and blunt) probably also involving dorsal column transmission. ${ }^{17}$ We found similar differences in the sensory level detected by the EPT and light touch and between the EPT and the pin prick, suggesting that if even the injury damaged sensory pathways to a different extent it is less likely that this factor largely contributed to our results. It is also less likely that these differences were due to asymmetries in the injury, because the EPT and ISNCSCI light touch and pin prick exams detected asymmetries in the majority of our SCI participants. The presence of some spared sensory function below the ISNCSCI sensory level highlights the lack of sensitivity of the ISNCSCI scoring system compared with the EPT exam. Indeed, ISNCSCI sensory exam is able to detect some spared sensory function, which gets lost during the quantification procedures. This might be in part related to the three-point nature of the numerical scoring system, where 2 is normal (just like sensation on the face), 1 is abnormal (different from the face, either hyposensitive 
or hypersensitive) and 0 is either absent or unable to tell the difference between sharp and dull more than $80 \%$ of the time, which does not allow a precise delineation of the preserved sensory function after a
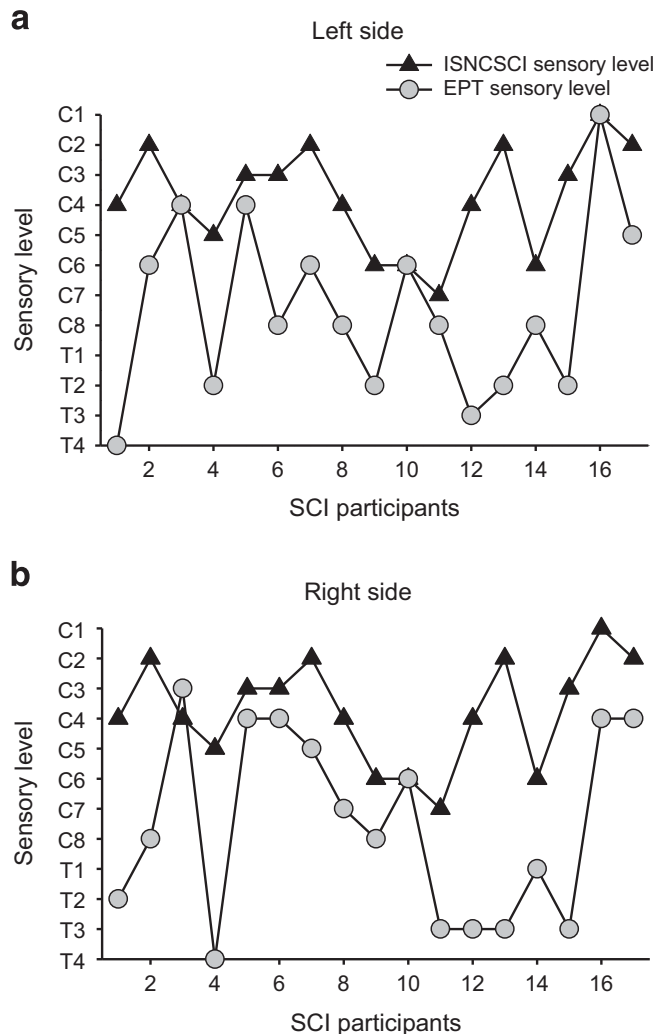

Figure 5 EPT vs ISNCSCI in SCl participants. In the graphs, the abscissa shows the SCl participants tested, and the ordinate shows the sensory level detected by the EPT (gray circles) and ISNCSCl (black triangles) exams in the left (a) and right (b) side of the body. Note that, regardless of the side tested, the sensory level detected by the EPT was below the sensory level detected by the ISNCSCI exam in the majority of $\mathrm{SCl}$ participants.
SCI, even though measurements can be reproduced over time. This might also be influenced by the restrictions imposed by the ISNCSCI sensory exam to define normality. As a 'normal' sensory level corresponds to the most caudal segment at which both sides of the body show a score of 2, this immediately excludes the possibility of detecting asymmetries and does not consider possible variations between light touch and pin prick scores. Thus, as proposed before, ${ }^{3-6}$ our results support the view that additional and more sensitive outcome measurements of sensory function are needed to supplement the ISNCSCI exam. Although, it is unclear whether discrepancies between EPT and ISNCSCI sensory level will be more or less pronounced in the acute and chronic phases of SCI, our study highlights the need to examine EPTs in a longitudinal manner in future studies.

Our results in control subjects agree with previous studies showing that EPT values are higher in males compared with females, ${ }^{8,15,18}$ which is congruent with evidence showing that females had lower

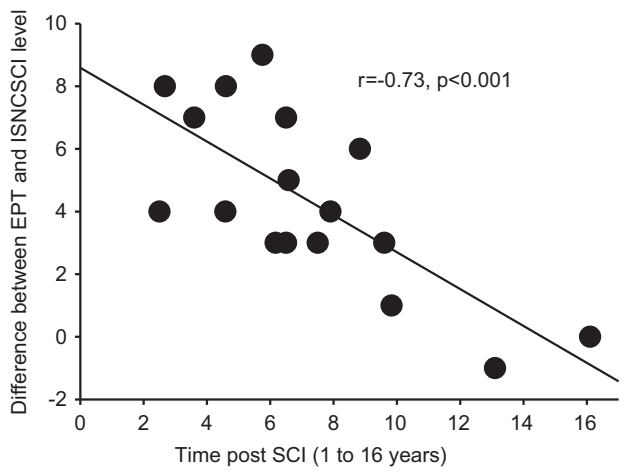

Figure 7 Correlation. The abscissa shows the number of years that individuals had $\mathrm{SCl}$, and the ordinate shows the difference between the sensory level detected by the EPT and the ISNCSCI sensory exams. Note that subjects with longer times with $\mathrm{SCl}$ showed a smaller difference between EPT and ISNCSCl sensory level. Note that the correlation was significant even though participants with $\mathrm{SCl}$ for more than 10 years were removed from the analysis $(r=-0.52, P=0.04)$.
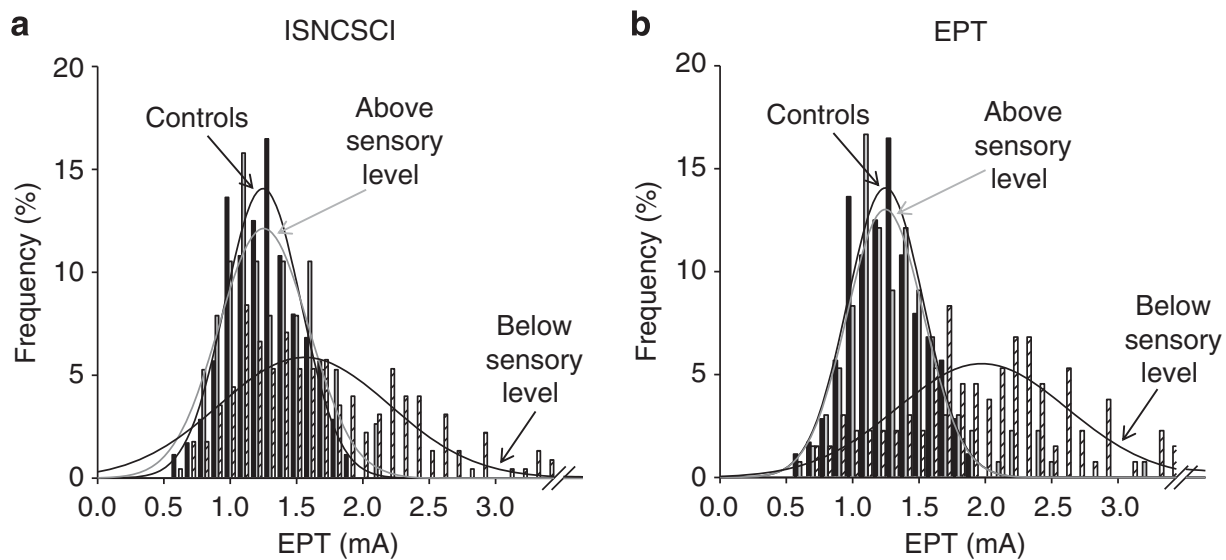

Figure 6 EPT frequency histograms above and below the detected sensory level. In the graphs, the abscissa shows the stimulus intensity (mA) used during testing, and the ordinate shows the number of times that the intensities were repeated, regardless of the dermatome tested expressed as a \% when the sensory level was detected by the ISNCSCI (a) and EPT (b) exams. In both graphs, the fitted lines show the frequency distribution of EPT values for old males (controls, indicated by an arrow), EPT values for dermatomes located above (indicated by gray arrow) and below (indicated by black arrow) the sensory level in SCI participants. Note that, in dermatomes above the sensory level detected by the ISNCSCI and EPT, the frequency distribution of EPT values was similar between $\mathrm{SCl}$ participants and controls but impaired in dermatomes below the detected sensory level. 
sensory thresholds to electrical and mechanical stimuli applied to the skin compared with males. ${ }^{19}$ We also found no differences in EPTs between left and right sides of the body as previously shown. ${ }^{8,20}$ On the other hand, our finding that older males showed higher EPT values compared with younger males and that this age difference was not found among females contrasts with earlier results showing no age-related difference in EPT among males ${ }^{20}$ and that women had higher EPT with age. ${ }^{20}$ These discrepancies with previous studies may be explained by the small number of subjects previously tested and by the lack of correction for multiple comparisons in previous studies. ${ }^{20}$ Our results underline the need to use proper age and gender comparable controls groups for comparisons of EPT values across populations.

In summary, our results extend previous findings showing that the EPT exam has good sensitivity to detect subclinical sensory changes that were not detected by the ISNCSCI sensory exam and suggest that in the chronic phase of incomplete cervical SCI, the EPT might provide a more sensitive tool to examine the recovery of sensory function.

\section{DATA ARCHIVING}

There were no data to deposit.

\section{CONFLICT OF INTEREST}

The authors declare no conflict of interest.

1 Herrmann KH, Kirchberger I, Biering-Sørensen F, Cieza A. Differences in functioning of individuals with tetraplegia and paraplegia according to the International Classification of Functioning, Disability and Health (ICF). Spinal Cord 2011; 49: 534-543.

2 Kirshblum SC, Burns SP, Biering-Sorensen F, Donovan W, Graves DE, Jha A et al. International standards for neurological classification of spinal cord injury (revised 2011). J Spinal Cord Med 2011; 34: 535-546.

3 Boakye M, Harkema S, Ellaway PH, Skelly AC. Quantitative testing in spinal cord injury: overview of reliability and predictive validity. J Neurosurg Spine 2012; 17: 141-150.
4 Steeves JD, Lammertse D, Curt A, Fawcett JW, Tuszynski MH, Ditunno JF et al. Guidelines for the conduct of clinical trials for spinal cord injury (SCI) as developed by the ICCP panel: clinical trial outcome measures. Spinal Cord 2007; 45: 206-221.

5 Ellaway PH, Kuppuswamy A, Balasubramaniam AV, Maksimovic R, Gall A, Craggs MD et al. Development of quantitative and sensitive assessments of physiological and functional outcome during recovery from spinal cord injury: a clinical initiative. Brain Res Bull 2011; 84: 343-357.

6 Alexander MS, Anderson KD, Biering-Sorensen F, Blight AR, Brannon R, Bryce TN et al. Outcome measures in spinal cord injury: recent assessments and recommendations for future directions. Spinal Cord 2009; 47: 582-591.

7 Rendell MS, Katims JJ, Richter R, Rowland F. A comparison of nerve conduction velocities and current perception thresholds as correlates of clinical severity of diabetic sensory neuropathy. J Neurol Neurosurg Psychiatry 1989; 52: 502-511.

8 Davey NJ, Nowicky AV, Zaman R. Somatopy of perceptual threshold to cutaneous electrical stimulation in man. Exp Physiol 2001; 86: 127-130.

9 Savic G, Bergstrom EM, Frankel HL, Jamous MA, Ellaway PH, Davey NJ. Perceptual threshold to cutaneous electrical stimulation in patients with spinal cord injury. Spinal Cord 2006; 44: 560-566.

10 Savic G, Frankel HL, Jamous MA, Jones PW, King NK. Sensitivity to change of the cutaneous electrical perceptual threshold test in longitudinal monitoring of spinal cord injury. Spinal Cord 2011; 49: 439-444.

11 Kramer JL, Moss AJ, Taylor P, Curt A. Assessment of posterior spinal cord function with electrical perception threshold in spinal cord injury. J Neurotrauma 2008; 25: 1019-1026.

12 King NK, Savic G, Frankel H, Jamous A, Ellaway PH. Reliability of cutaneous electrical perceptual threshold in the assessment of sensory perception in patients with spinal cord injury. J Neurotrauma 2009; 26: 1061-1068.

13 Lauschke JL, Leong GW, Rutkowski SB, Waite PM. Changes in electrical perceptual threshold in the first 6 months following spinal cord injury. J Spinal Cord Med 2011; 34: 473-481.

14 Cortes M, Elder J, Rykman A, Murray L, Avedissian M, Stampa A et al. Improved motor performance in chronic spinal cord injury following upper-limb robotic training. NeuroRehabilitation 2013; 33: 57-65.

15 Leong GW, Lauschke J, Rutowski SB, Waite PM. Age, gender, and side differences of cutaneous electrical perceptual threshold testing in an able-bodied population. J Spinal Cord Med 2010; 33: 249-255.

16 Dimitrijevic MR, Kakulas BA, McKay WB, Vrbova G. Restorative Neurology of Spinal Cord Injury. Oxford University Press: New York, NY, 2011.

17 Vasquez N, Gall A, Ellaway PH, Craggs MD. Light touch and pin prick disparity in the International Standard for Neurological Classification of Spinal Cord Injury (ISNCSCI). Spinal Cord 2013; 51: 375-378.

18 Maffiuletti NA, Herrero AJ, Jubeau M, Impellizzeri FM, Bizzini M. Differences in electrical stimulation thresholds between men and women. Ann Neurol 2008; 63: 507-512.

19 Torgén M, Swerup C. Individual factors and physical work load in relation to sensory thresholds in a middle-aged general population sample. Eur J App/ Physiol 2002; 86: 418-427.

20 Leong GW, Gorrie CA, Ng K, Rutkowski S, Waite PM. Electrical perceptual threshold testing: a validation study. J Spinal Cord Med 2009; 32: 140-146. 